\title{
Rotation, activity, and stellar obliquities in a large uniform sample of Kepler solar analogs
}

\author{
Derek Buzasi ${ }^{1}{ }^{*}$, Andy Lezcano ${ }^{1}$, and Heather L. Preston ${ }^{2}$ \\ 1 College of Arts and Sciences, Florida Gulf Coast University, Fort Myers, FL 33965, USA \\ *Corresponding author: dbuzasi@fgcu.edu \\ 2 Calusa Nature Center \& Planetarium, 3450 Ortiz Ave., Fort Myers, FL 33905, USA
}

Received 15 February 2016 / Accepted 1 September 2016

\begin{abstract}
In this study, we undertook a deep photometric examination of a narrowly-defined sample of solar analogs in the Kepler field, with the goals of producing a uniform and statistically meaningful sample of such stars, comparing the properties of planet hosts to those of the general stellar population, and examining the behavior of rotation and photometric activity among stars with similar overall physical parameters. We successfully derived photometric activity indicators and rotation periods for 95 planet hosts (Kepler objects of interest [KOIs]) and 954 solar analogs without detected planets; 573 of these rotation periods are reported here for the first time. Rotation periods average roughly $20 \mathrm{~d}$, but the distribution has a wide dispersion, with a tail extending to $P>35 \mathrm{~d}$ which appears to be inconsistent with published gyrochronological relations. We observed a weak rotation-activity relation for stars with rotation periods less than about $12 \mathrm{~d}$; for slower rotators, the relation is dominated by scatter. However, we are able to state that the solar activity level derived from Virgo data is consistent with the majority of stars with similar rotation periods in our sample. Finally, our KOI sample is consistently approximately 0.3 dex more variable than our non-KOIs; we ascribe the difference to a selection effect due to low orbital obliquity in the planet-hosting stars and derive a mean obliquity for our sample of $\chi=6_{-6}^{+5^{\circ}}$, similar to that seen in the solar system.
\end{abstract}

Key words. Stars: activity - Stars: solar-type - Stars: rotation

\section{Introduction}

The Kepler mission has provided an unparalleled window into the nature and occurrence rate of planets around other stars (Borucki et al. 2010), with 4,743 planet candidates and 1,042 confirmed exoplanets identified as of this writing. This unprecedented flood of information was enabled by the mission's sub-millimagnitude photometric precision combined with its $\sim 100$ square degree field of view. As a necessary part of Kepler's exoplanet program, the spacecraft observed approximately $2 \times 10^{5}$ stars, and these constitute a large uniform stellar sample ideal for improving our insights into stellar activity, rotation, and granulation across the HR diagram. We note that in the context of this paper, unless noted otherwise we use "activity" as a synonym for "photometric variability".

Data from both CoRoT and Kepler have been used by a number of different researchers to examine rotation periods, with results that have been intriguing although not always consistent. Affer et al. (2012) examined the CoRoT sample and found a double-peaked distribution for their 1,727 rotation periods, ascribing the short-period peak $(P<10 \mathrm{~d})$ to young stars and binaries and the long-period peak $(P>35 \mathrm{~d})$ to older stars, with a fall-off in the distribution only occurring for $P>80$ d. Walkowicz \& Basri (2013) examined rotation periods and activity levels for nearly 1,000 Kepler objects of interest (KOIs), using only data from Quarter 9, using the rotational periods to derive ages via gyrochronology. They found a wide range in photometric amplitude for a given Rossby number, implying significant variations in spot number and distribution as a function of time, and a weak relationship between photometric range and rotational period (their Fig. 2). García et al. (2014) took a different approach, examining only Kepler stars with asteroseismic detections; these were necessarily bright, and thus only 78 were cool dwarfs with detectable rotation periods. The majority of these $(61.5 \%)$ had activity levels within the solar range, and García et al. (2014) were able to fit a Skumanich-type spindown relation with a power law index of 0.51 to a subsample of the bestcharacterized 15 cool dwarfs. do Nascimento et al. (2014) focused on solar analogs and twins, examining 75 candidates from the Kepler field and identifying 34 as solar analogs. This sample had rotation periods ranging from 6 to $30 \mathrm{~d}$, with a mean of $19 \mathrm{~d}$; typical rotational uncertainties were $10-15 \%$ of the measured rotation periods. The largest extant sample was examined by McQuillan et al. (2014) who used an autocorrelation technique to derive rotation periods for 34,030 of the Kepler targets, finding a general trend of decreasing rotation period with increasing mass, with most "solarlike" stars (their 5,500< $<T_{\text {eff }}<6,000$ bin) showing periods of 10-25 d. Interestingly, McQuillan et al. (2014), found a significant number of Kepler stars in this effective temperature bin with rotation periods as short as $1 \mathrm{~d}$ or less. Nielsen et al. (2013) and Reinhold et al. (2013) examined similarly broad samples of the Kepler database, with similar results.

We do not attempt to duplicate all of this prior work. Instead, we focus on a particular astrophysically interesting subset of stars, the solar analogs. Two complementary approaches have historically been adopted for studies of the Sun as a star: the first centers on in-depth studies of the 
Sun itself, and the second on the study of solar analogs, stars which are like the Sun and thus might shed light on characteristics of its composition, structure, activity, planetary system, evolution, and future. Solar analogs are useful in many ways, ranging from finding night-time proxies for the Sun for calibration purposes to their use as snapshots of the evolutionary history of the Sun. Solar analog studies have helped to establish that chromospheric activity is key to understanding stellar rotation, activity, and dynamos. More than 40 years ago, Skumanich (1972) noted the decline of chromospheric activity in cool stars with increasing age. Skumanich used the near-UV Ca II H and K lines as activity diagnostics and proxies for surface magnetic field strength and filling factor, as do the various more recent ground-based monitoring programs (such as the Mt. Wilson HK project and the Lowell SSS; Baliunas \& Jastrow 1990; Baliunas et al. 1995; Hall et al. 2007). There is some evidence that the Sun itself is relatively metal-rich (Rocha-Pinto \& Maciel 1996), lithium-depleted (Takeda 2007), and deficient in chromospheric activity (Hall et al. 2007, 2009) relative to its putative analogs, but for many of these claims there is conflicting evidence (see, e.g., Hall et al. 2009; Adibekyan et al. 2013) and so it is in some ways unclear, as some authors have put it, whether or not the Sun itself would qualify as a good solar twin!

An additional area of interest lies in the activity level of the Sun compared to its peers. While some researchers have argued that the Sun is depressed in activity relative to inactive solar analogs (see, e.g. Radick et al. 1998; Hall et al. 2007), others suggest that the Sun does not appear to have unusually low photometric variability compared with these stars (Hall et al. 2009). Giampapa et al. (2006) found that the range of $\mathrm{Ca}$ II $\mathrm{H}+\mathrm{K}$ chromospheric activity levels seen in the Sun during its activity cycle significantly overlaps the range seen in solar-like stars in the open cluster M67, which is similar to the Sun in both age and metallicity, while Önehag et al. (2011) performed a differential spectroscopic analysis of a solar analog in the same cluster and found it to be nearly indistinguishable from the Sun. Together, these imply a fundamental similarity between the variability of the Sun and other stars of similar age and composition, though clearly research into a broader group of solar analogs is required to confirm this.

Baliunas \& Jastrow (1990) found that nearly $1 / 3$ of solartype stars showed appreciably lower chromospheric activity levels than the Sun, which they ascribed to these stars being in Maunder minima states. However, later research (Hall et al. 2009; Takeda et al. 2012) failed to corroborate this finding, and Hall et al. (2009) presented evidence that Ca II $\mathrm{H}-\mathrm{K}$ indices were not a good discriminant of this state, in any case. Wright (2004) suggested that the majority of "Maunder minimum" identifications were actually of more evolved stars, and "there is no unambiguous identification of another star in a Maunder minimum state". At the very least, such states appear to be rare (see also Lubin et al. 2010, 2012), so we must identify and observe a large number of solar analogs in order to unambiguously detect any.

A large part of the difficulty in placing the Sun in context among its analogs arises from the simple fact that the catalog of good solar analogs is so small. Perhaps the most complete extant survey is Porto de Mello et al. (2014), who examined 133 candidates in a survey which was complete to $40 \mathrm{pc}$ and partially complete to $50 \mathrm{pc}$, roughly corresponding to $\mathrm{V}<8.5$. Their definition of "solar twin" was substantially similar to our definition of solar analog (see their Fig. 3 and our criteria below), and was first defined by constraints on $T_{\text {eff }}$ $( \pm 65 \mathrm{~K}), \quad[\mathrm{Fe} / \mathrm{H}] \quad( \pm 0.14 \mathrm{dex}), \quad$ and $\log g \quad( \pm 0.2 \mathrm{dex})$, supplemented, where available, by high-resolution spectroscopy. Stellar chromospheric activity levels were estimated using $\mathrm{Ca}$ II $\mathrm{H}$ and $\mathrm{K}$ or $\mathrm{H} \alpha$ fluxes, though photometric activity was not considered. Out of this large initial sample, after detailed examination, only 30 stars remained. Similar results were obtained through searches of the Geneva-Copenhagen survey by Datson et al. (2015), through high-resolution Subaru spectroscopy by Takeda et al. (2012), and through contemporaneous photometry and spectroscopy by Hall et al. (2009). The bottom line: we simply lack a large, coherent, consistent sample of solar analogs.

By adopting a focus on solar analogs in the Kepler field, we have three goals: first, to take advantage of the Kepler data set to examine a unique and uniform sample which possesses enough members to better enable significant conclusions to be drawn; second, to address some of the inconsistencies mentioned above; and third, to carry out an in-depth photometric comparison of known exoplanet host stars to the general population. An additional advantage of focusing on solar analogs, narrowly defined, is that we are able to examine the behavior of rotation and activity without the potentially confusing inclusion of color- or mass-dependent factors, as our stars all have these parameters in common.

\section{Data analysis}

Our intention was to sample only potential solar analogs. Accordingly, we began with stars in the updated Kepler Input Catalog (Brown et al. 2011; Huber et al. 2014) with $\mathrm{K}_{\text {mag }}<14$ and removed all known eclipsing binaries according to the catalog of Prša et al. (2011), as updated on the Villanova Eclipsing Binary website (http://keplerebs.villanova.edu/). In order to increase our sample of planet-hosting stars, we extended our magnitude limit for these to $\mathrm{K}_{\mathrm{mag}}<16$. We then restricted our search to stars with $\left|T_{\text {eff }}-T_{\odot}\right|<100 \mathrm{~K}$, where we adopted the revised effective temperature scale due to Huber et al. (2014) and Pinsonneault et al. (2012), and $T_{\odot}=5,772 \mathrm{~K}$ (Mamajek et al. 2015). In addition, we required $-0.3<[\mathrm{Fe} / \mathrm{H}]<0.3$ and $4.2<\log g<4.6$ per Huber et al. (2014). The motivation for these ranges is that they approximately sample the solar values within the quoted $\pm 1 \sigma$ uncertainties for each parameter. Targets with missing values for any of these parameters were eliminated from our sample. Finally, we split the sample into KOIs and non-KOIs, based on the KOI list from the NASA Exoplanet Archive (Akeson et al. 2013; http://exoplanetarchive.ipac.caltech.edu). Statistically, the vast majority (Fressin et al. 2013; Coughlin et al. 2014) of the KOIs are expected to turn out to be true planet hosts rather than false positives, and for the purposes of this study we treated this sample as if all were truly exoplanet host stars. The non-KOI sample, of course, is expected to contain stars both with and without exoplanets, whether due to geometric misalignment leading to the lack of visible eclipses or to other reasons, such as small transit amplitude or long orbital period. Once we had established the input list in this way, we further qualified it by eliminating stars with fewer than two quarters of Kepler data. Our final sample consisted of 269 Kepler Objects of Interest and 2011 non-KOI stars. Twenty-seven of our KOIs host Jupiter-sized planets, defined as $\mathrm{R}_{\mathrm{PL}}>6 \mathrm{R}_{\oplus}$. For each star, we downloaded from Mikulski Archive for Space Telescopes (MAST; https://archive.stsci.edu/kepler/) 
the complete long-cadence Kepler data set, relying on the PDC-MAP algorithm (Smith et al. 2012) to remove instrumental effects. We then preprocessed each quarter by removing any remaining instrumental glitches and applying a simple linear fit over the entire quarter to remove the lowestfrequency noise.

We searched for rotation periods in our sample using a discrete Fourier transform (DFT) algorithm to search the region [2 d, $44 \mathrm{~d}$ ], identifying the rotation period as the largest amplitude spectrum peak found in that region. To avoid the necessity of "stitching" the separate quarters together, with the concomitant possibility of introducing artificial periodicities at $90 \mathrm{~d}$ and aliases thereof, we treated each quarter independently. Most of our targets had 17 quarters (or partial quarters) of data, though we did not necessarily disqualify those for which this was not true. In each quarter, we estimated the signal-to-noise ratio (SNR) of the peak by comparing the peak value to the high-frequency noise level in the data. We then formed an overall rotation period estimate for each star by calculating a weighted mean of the periods from the different quarters, with weights for each quarter determined by the SNR and quarters with SNR $<10$ rejected. We also rejected stars with fewer than six quarters of data meeting our criteria. We similarly estimated the uncertainties in the rotation periods from the weighted standard deviation of the values for the individual quarters, which generally resulted in more conservative error estimates than would have been derived from other criteria, such as widths of the peaks in the power spectra. Our motivation for adopting this technique was the desire to obtain rotation period measurements for fainter stars in the sample, recognizing that the tradeoff was likely to be larger errors on those measurements.

In the case of KOIs, we removed transits from the time series prior to analysis, in order to avoid the possible contamination of our rotation period measurements by the presence of transits. Excision was achieved by first identifying the transits based on data from the Exoplanet Archive (http:// exoplanetarchive.ipac.caltech.edu/), then removing points lying in or near the time of transit (a window centered on the transit and 1.2 times its length was used), and finally replacing the excised points with points interpolated using a shape-preserving piecewise cubic interpolation based on the surrounding time series.

In order to assure ourselves that this procedure adequately removed the transit signals without introducing new contaminants, we conducted a series of simulations. In these, we took our original complete sample of non-KOI stars and introduced into each of these light curves artificial transits (simple step functions) with rotation periods and depths randomly chosen from within the range of the KOI sample. These artificial transits were then removed and the gaps interpolated using the same procedure we used on the KOIs; the resulting period measurements were compared to those determined prior to artificial transit insertion. We repeated this procedure 10 times for each quarter of each star, and Figure 1 shows the results. Approximately $99.4 \%$ of relative rotation period errors are smaller than $1 \%$, and there are no significant biases introduced. While the process clearly leads to some additional uncertainty in our rotation period determinations, that additional error is extremely small compared to the 10-20\% uncertainties we typically find in our rotation period determinations (see below). Accordingly, we have confidence in adopting our procedure to measure periods for the KOIs in our sample.

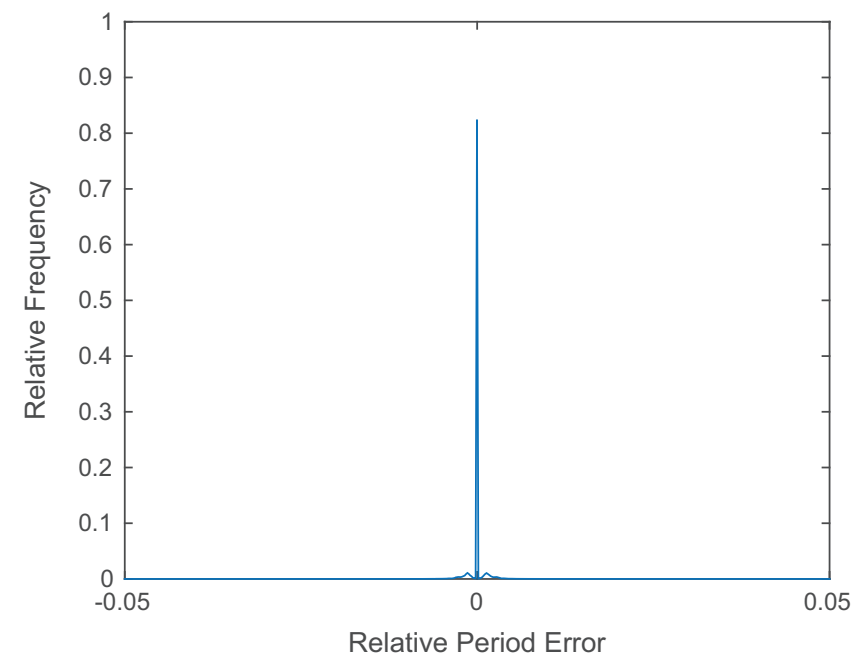

Fig. 1. The distribution of relative rotation period errors introduced into our suite of test light curves by our transit removal procedure; more than $99 \%$ of introduced errors are less than $1 \%$.

Table 1. Solar Virgo $g+r$ results.

\begin{tabular}{lccc}
\hline \hline & $P_{\text {rot }}(\mathrm{d})$ & $\sigma_{\mathrm{P}}(\mathrm{d})$ & Range $(\mathrm{ppt})$ \\
\hline Solar minimum & 28.5 & 2.3 & 0.23 \\
Solar maximum & 27.8 & 2.5 & 0.93 \\
\hline
\end{tabular}

We then tested our period-finding procedure using solar data, inspired by the approach outlined in Basri et al. (2011). In particular, we chose 2 four-year segments of SOHO Virgo $g+r$ data to sample the Sun around both solar maximum and solar minimum. The former segment began in March 2000 and the latter in March 2006; both were broken into 16-quarter segments and processed in the same manner as our Kepler data. Results are summarized in Table 1, which also shows the derived photometric range $R$ for each time series (as defined by Basri et al. 2011 and in Sect. 2); in both cases, we successfully recovered the solar rotation period. Interestingly, Nielsen et al. (2013) applied a similar approach and failed to reliably recover the solar rotation, while Basri et al. (2011) found only that $P_{\odot} \sim 20 \mathrm{~d}$, primarily due to their use of only one quarter of data. Both pointed out that, as variability is expected to decrease as rotation period increases, such failure implies a bias in that stars with long rotation periods are less likely to be detected. The "hare-and-hounds" exercise in Aigrain et al. (2015) performed a similar test with solar data, and our results are comparable to the better-performing algorithms in that paper. As the Sun is a relatively low-activity star in comparison to our overall sample (see Sect. 3.1), our ability to recover its rotation period in this way reinforces our confidence in our algorithm.

Motivated by this finding, we next conducted numerical experiments in an attempt to discover the limitations of our period-finding approach in the Kepler sample. We constructed model stars with 1-3 spot groups located at latitudes randomly selected between 0 and $40^{\circ}$. Initial spot groups were sized to produce a maximum photometric amplitude based on the empirical fit of Hartman et al. (2009), and spot positions evolved according to a solar differential rotation model. Spot group sizes were allowed to evolve according to a linear growth and decay model chosen to emulate the results of Gafeira et al. (2014). We then added noise based on the 
(a)

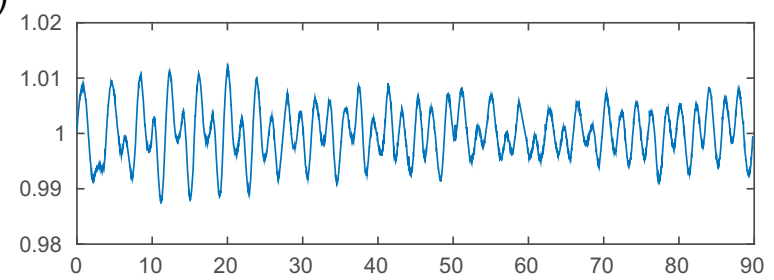

(c)

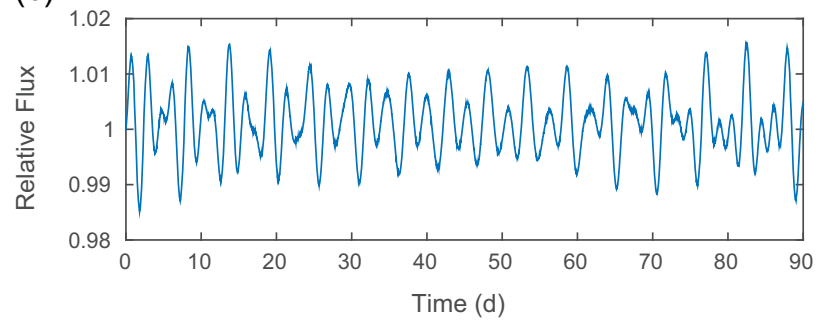

(b)

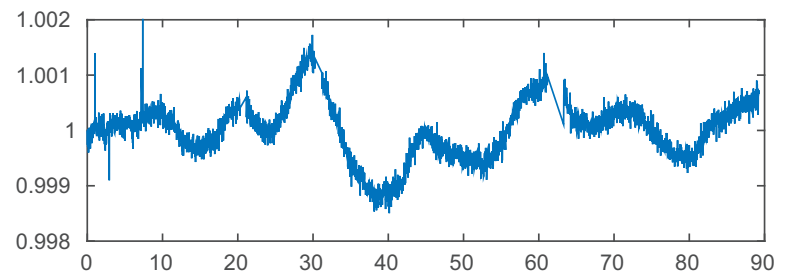

(d)

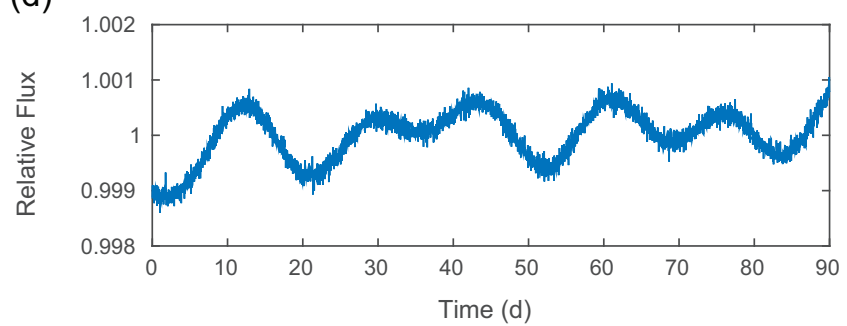

Fig. 2. An illustration of the output from our synthetic spot model light curve generator, used to validate our period-finding approach. (a) Compares a one-quarter light curve for a real star with a $3.8 \mathrm{~d}$ period (KIC 4912088, Quarter 6) with (c) model output for a star with similar rotation period and Kepler magnitude. (b, d) Repeats the comparison for a star with a 28 d period (KIC 7269388, Quarter 3).

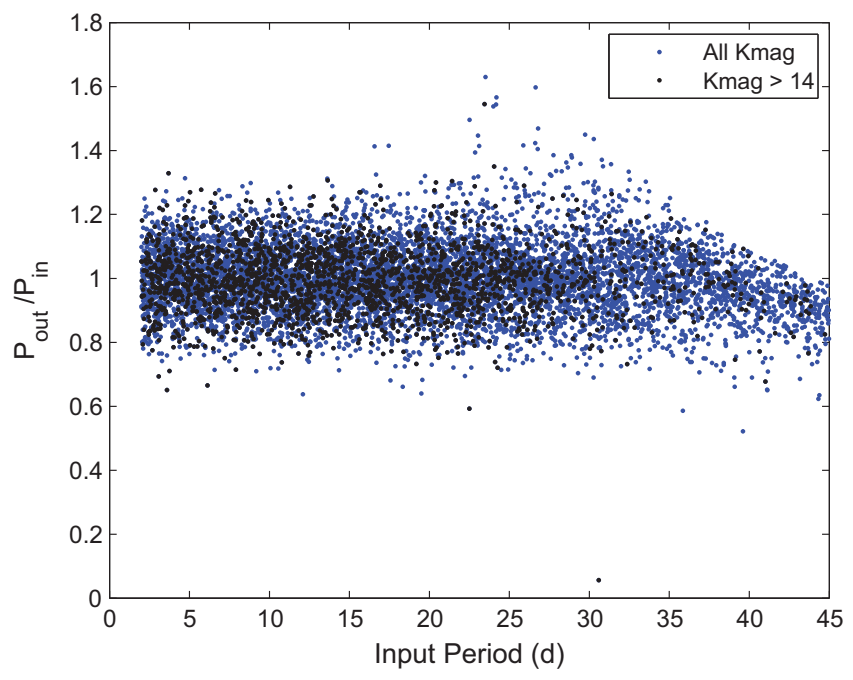

Fig. 3. The results of our period-finding algorithm applied to an array of model stars with a range of magnitudes from 10 to 16 and noise from Gilliland et al. (2011). Artificial spots were added as described in the text. While period recovery begins to suffer for $P>30 \mathrm{~d}$, errors are typically $<20 \%$ for $P<40 \mathrm{~d}$, even for the fainter stars in our sample ( $\mathrm{K}_{\text {mag }}>14$, shown in black).

findings of Gilliland et al. (2011) for Kepler, including solar granulation noise. Figure 2 shows some representative light curves from our simulator and compares them to real light curves from the Kepler database.

We examined 90-day segments of $10^{5}$ of the resulting light curves for a range of rotation rates from 2 to 40 days and a range of stellar magnitudes from 10 to 16 . A sample of results from these simulations is shown in Figure 3. We were able to successfully recover rotation periods with relative errors $<10 \%$ for all stars brighter than $\mathrm{K}_{\operatorname{mag}}=14$, while errors grew to $\sim 20 \%$ for the longest-period stars with $K_{\text {mag }}=16$. Some systematic errors begin to occur at periods beyond $30 \mathrm{~d}$, but these are small compared to other error sources for all stars in our sample. We conclude that our derived rotation periods are reliable out to approximately 40 days for solar-like stars in the Kepler field.

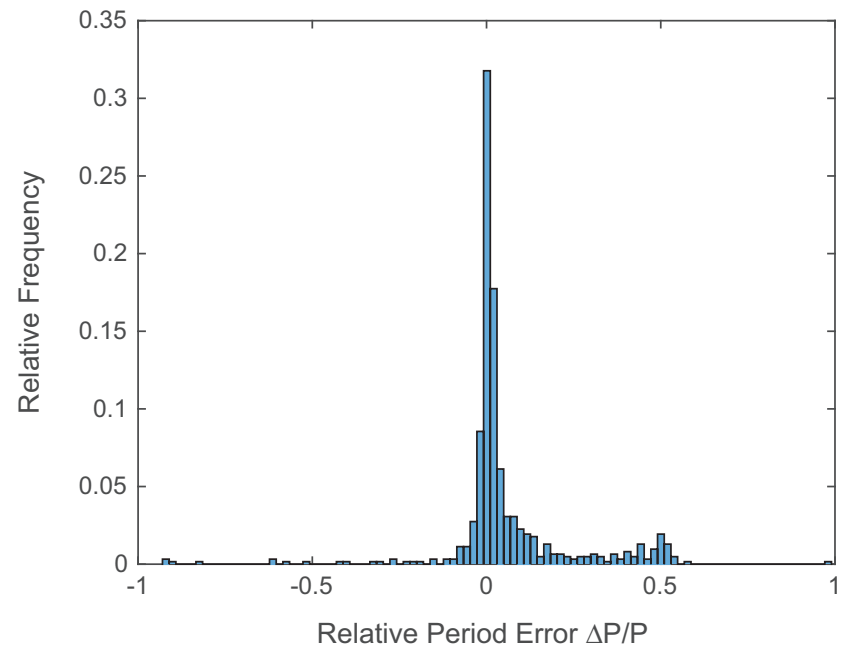

Fig. 4. A histogram of the relative differences between the rotational periods derived in this work and those previously derived by McQuillan et al. (2014) for targets with SNR > 10 appearing in both works. Differences are in the sense of $P-P_{\text {McQuillan }}$, and the standard deviation of the central peak is $6.9 \%$. The secondary peak is due to stars for which our period is twice as long as that reported by McQuillan et al. (2014); see text for further discussion.

An additional test of our methodology derives from a comparison to the published rotation periods of McQuillan et al. (2014). In Figure 4, we show the relation between our rotation period and that derived by McQuillan et al. (2014) for all 451 targets with detected periods in both works. For the majority of stars we both find similar periods (the standard deviation of the central peak is $6.9 \%$ ), comparable to the typical internal errors we estimated earlier. However, there are two populations for which our rotation periods differ substantially. The first is a small number of stars for which McQuillan et al. (2014) report very short periods, typically $<2 \mathrm{~d}$. Individual inspection shows these to be misidentified as solar analogs (they are typically eclipsing binaries or other variable stars), and we have rejected these from our sample. The second, somewhat larger group, shown in Figure 4, centered around 0.5 , represents stars for which we report 
periods twice as long as those in McQuillan et al. (2014). We have examined these light curves individually, and find it impossible to determine the "correct" period manually, so we have retained our values for these, noting that their number is small enough not to bias any of the conclusions in this work.

We have also applied our algorithm to the "hare-andhounds" stellar data set created by Aigrain et al. (2015) and used by them to test a range of different period-finding methods. Aigrain et al. (2015) simulated 1,000 d time series, which differ from the total length of the Kepler observations $(\sim 1,600 \mathrm{~d})$, and which simulate rotation periods from 1 to 50 days. Accordingly, we broke their light curves into 10 approximately 90-day segments, and examined only the 699 (of 770) of the "noisy" simulations that had input periods within the 2-44 day range we search. We found we recovered the input rotation periods in $74 \%$ of cases; of these, $67 \%$ were "good" and $87 \%$ "OK", by the criteria in Aigrain et al. (2015). Comparison to Table 3 in Aigrain et al. (2015) shows that our recovery rates are broadly comparable to those of other methods. Unfortunately, the nature of the data set does not allow us to meaningfully compare our recovery rate for low-amplitude slow rotators to those of other approaches, as the test data were generated without the use of a rotationactivity relation, and our artificial quarter "breaks" lack the instrumental effects typical of real Kepler data. However, based on our earlier simulations, we have confidence that our methodology performs well for such cases, at the cost of increased uncertainty in rotation period determination. Figure 5 shows our relative internal errors $\sigma_{\mathrm{Per}}$, derived from the weighted mean of the standard deviation of results from the individual quarters. The mean of this distribution is $6.5 \%$, consistent with our simulations reported above. We note that our approach is clearly not superior for all cases; for example, ACF-based approaches are more efficient for detecting rotation in the first place, and all of the alternate methods in Aigrain et al. (2015) are superior for the detection of rotation periods greater than 44 days (which we would not detect at all).

In addition to the rotation period, we also calculated the photometric range $R$ for each time series. As defined by Basri et al. (2011), $R$ represents the range of variation between the 5 th and 95th percentile for each star. We follow the example of Bastien et al. (2013) to fit and subtract an envelope as a function of $\mathrm{K}_{\mathrm{mag}}$, as shown in Figure 6, to correct for the rising contribution to $R$ from fainter stars simply due to increasing photon noise. Our envelope fit takes the form

$$
\log R_{\mathrm{env}}=0.21+2.55 \times 2.512^{\mathrm{K}_{\mathrm{mag}}-16} .
$$

We experimented with varying the form (by adding higherpower terms in $\mathrm{K}_{\mathrm{mag}}$ ) and parameters of our envelope fit, and our conclusions are robust to such minor variations.

Tables 2 and 3 also give the resulting periods and ranges for our targets, as well as other stellar parameters. The "Quarters" column in the tables reflects the number of quarters used in forming the weighted means. The tables reflect the 95 KOIs (9 Jupiter-sized) and 954 non-KOIs for which our procedure reported rotation periods; 573 of these are targets for which no rotation period has previously been reported in the literature. This constitutes $46 \%$ of our original sample, which compares favorably with the $25.6 \%$ recovery rate of McQuillan et al. (2014) (though the latter from a much more broadly constituted sample), and is closer to the $\sim 70 \%$ rate typical of the hare-and-hounds exercises recently reported by

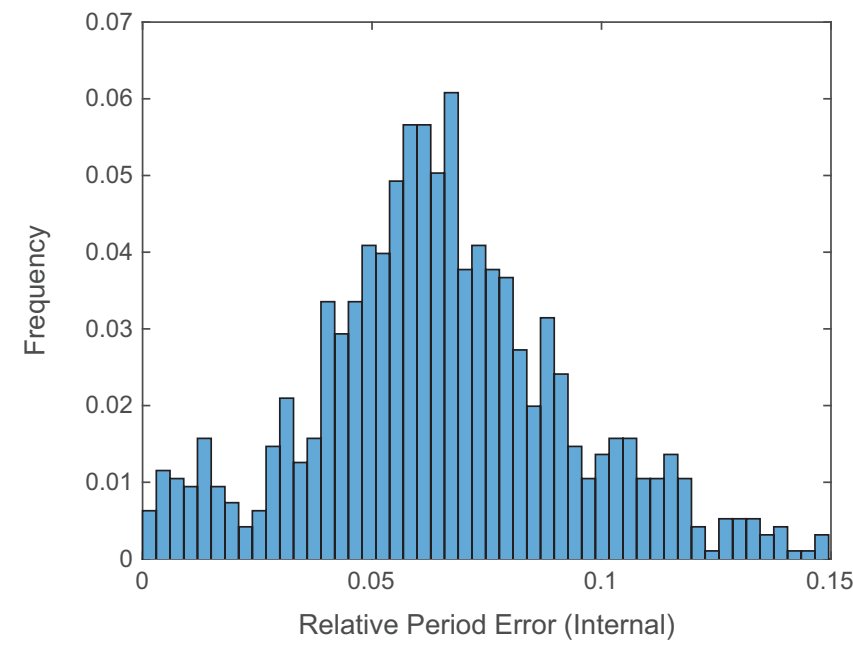

Fig. 5. The relative internal errors $\sigma_{\mathrm{P}}$ we report for our sample. The mean of the distribution is at $6.5 \%$, consistent with the simulation results reported earlier.

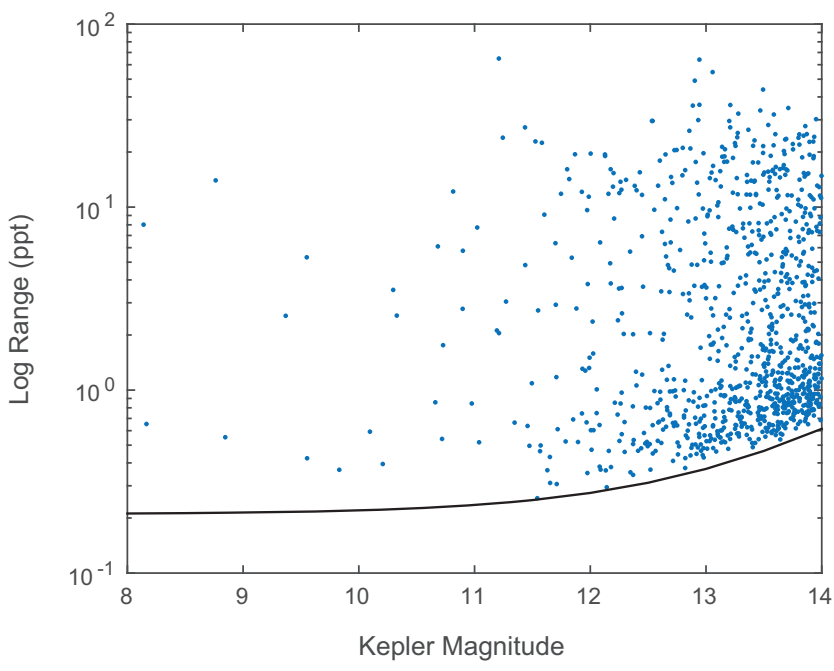

Fig. 6. The raw range measurements (in ppt) for our non-KOI sample, along with the lower envelope fit to that distribution (black line), which is subtracted following the procedure outlined in Bastien et al. (2013) to obtain the final range result $R$.

Aigrain et al. (2015). We note in passing that it is curious that recovery rates for actual data appear to be noticeably lower than those from synthetic data, though in the case of Aigrain et al. (2015), this may be due to the lack of any adopted rotation-activity relation, so that slow-rotating stars in the synthetic data were on average considerably more active than those in the real world. A simple check on biases in our procedures is to compare the cumulative frequency distributions (CFDs) for $T_{\text {eff }}$ between our final populations of KOIs and non-KOIs, as shown in Figure 7a. A Kolmogorov-Smirnov $(\mathrm{K}-\mathrm{S})$ test confirms the visual impression that there is no statistically significant difference apparent $(p=0.88)$ between the two groups. Figure $7 \mathrm{~b}$ shows the CFDs for $[\mathrm{Fe} / \mathrm{H}]$ for these two populations, and we find no significant difference (K-S $p=0.91$ ) between the two populations. We also compared the Jupiter-sized population to the non-Jupiters; though the former is very small, a K-S test shows no significant difference between these populations either (K-S $p=0.91)$. 
Table 2. Measured parameters for Kepler targets (KOIs).

\begin{tabular}{cccccccc}
\hline \hline $\mathrm{KIC}$ & $\mathrm{T}_{\text {eff }}(\mathrm{K})$ & $\log g$ & {$[\mathrm{Fe} / \mathrm{H}]$} & Range $(\mathrm{ppt})$ & $P_{\text {rot }}(\mathrm{d})$ & $\sigma_{\mathrm{P}}(\mathrm{d})$ & Quarters \\
\hline 1872,821 & 5,718 & 4.549 & -0.120 & 0.982 & 14.506 & 1.537 & 8 \\
3234,843 & 5,817 & 4.544 & -0.140 & 1.032 & 28.199 & 2.076 & 6 \\
3440,118 & 5,839 & 4.247 & -0.280 & 28.008 & 4.724 & 0.133 & 17 \\
3446,746 & 5,696 & 4.417 & 0.040 & 1.071 & 31.180 & 3.387 & 7 \\
3541,946 & 5,733 & 4.551 & -0.120 & 10.539 & 15.756 & 0.975 & 17 \\
\hline
\end{tabular}

Note. The complete version of the table is available in the electronic supplement.

Table 3. Measured parameters for Kepler targets (non-KOIs).

\begin{tabular}{cccccccc}
\hline \hline KIC & $T_{\text {eff }}(\mathrm{K})$ & $\log g$ & {$[\mathrm{Fe} / \mathrm{H}]$} & Range $(\mathrm{ppt})$ & $P_{\text {rot }}(\mathrm{d})$ & $\sigma_{\mathrm{P}}(\mathrm{d})$ & Quarters \\
\hline 1434,708 & 5,472 & 4.337 & 0.000 & 6.587 & 21.274 & 1.827 & 13 \\
1849,885 & 5,750 & 4.345 & -0.160 & 1.340 & 25.388 & 1.607 & 16 \\
1868,785 & 5,833 & 4.496 & 0.210 & 0.529 & 25.948 & 2.714 & 10 \\
1996,405 & 5,837 & 4.339 & -0.060 & 0.349 & 24.355 & 1.462 & 12 \\
2011,160 & 5,776 & 4.382 & 0.180 & 1.533 & 27.302 & 0.906 & 14 \\
\hline
\end{tabular}

Note. The complete version of the table is available in the electronic supplement.

(a)

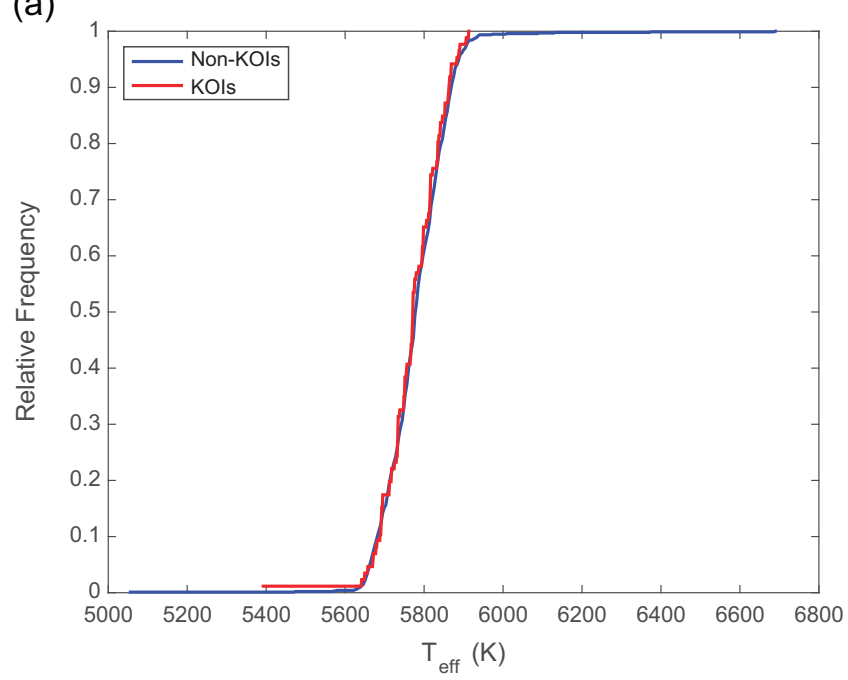

(b)

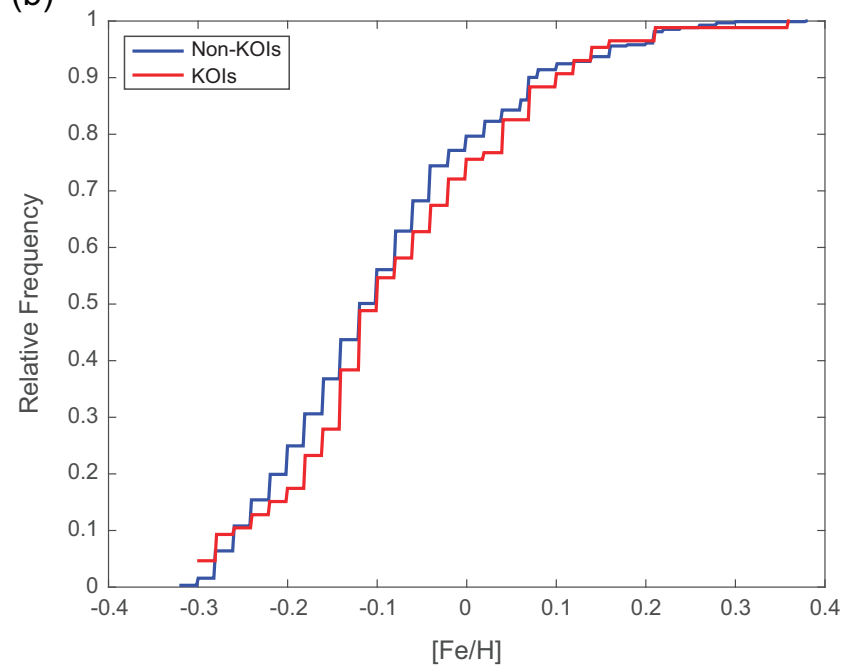

Fig. 7. Cumulative frequency distributions (CFDs) for (a) $T_{\text {eff }}$ and (b) $[\mathrm{Fe} / \mathrm{H}]$ confirm the consistency of our KOI and non-KOI samples.

\section{Results and discussion}

\subsection{Rotation and activity}

Figure 8 illustrates histograms for rotation rates for both the KOI and non-KOI samples. Mean KOI periods are $20.1 \pm 8.2 \mathrm{~d}$, not visually substantially different from the $21.6 \pm 8.9 \mathrm{~d}$ for the non-KOI sample, though a K-S test does show the KOI and non-KOI samples to differ at a level which is marginally significant $(p=0.01)$. Our rotation period distribution is similar to that reported by McQuillan et al. (2014) for stars in the same temperature range (correcting for the discrepancy between the Kepler Input Catalog [KIC] temperatures used by McQuillan et al. (2014) and those used in this work), though our new detections tend to be less active and slower rotating, so that the fall-off in number visible in their Figure 1 for periods greater than $25-30 \mathrm{~d}$ is here extended to $30-35 \mathrm{~d}$.
The distribution of activity levels, as determined by the photometric range parameter $R$, is shown Figure 9a for both the KOI and non-KOI samples. While the two distributions are similar in overall appearance, KOIs appear on average approximately 0.3 dex more variable than non-KOIs (K-S $\left.p<10^{-6}\right)$. The CFDs in Figure 9b illustrate this more clearly. We discuss our interpretation of the reason for this difference below in Section 3.3.

Figure 10 shows this result in a different form, plotting range as a function of rotational period. The Period-Range relation of Hartman et al. (2009), which was earlier used in our simulations, is also shown; while it provides a reasonable estimate of variability for rapid rotators, it clearly fails for periods longer than about a week. In addition, the rotationactivity level appears to have a break corresponding to periods of around $P=12 \pm 2 \mathrm{~d}$, indicated by the dotted vertical line, and clearly visible in the binned relationship. For solar analogs 
(a)

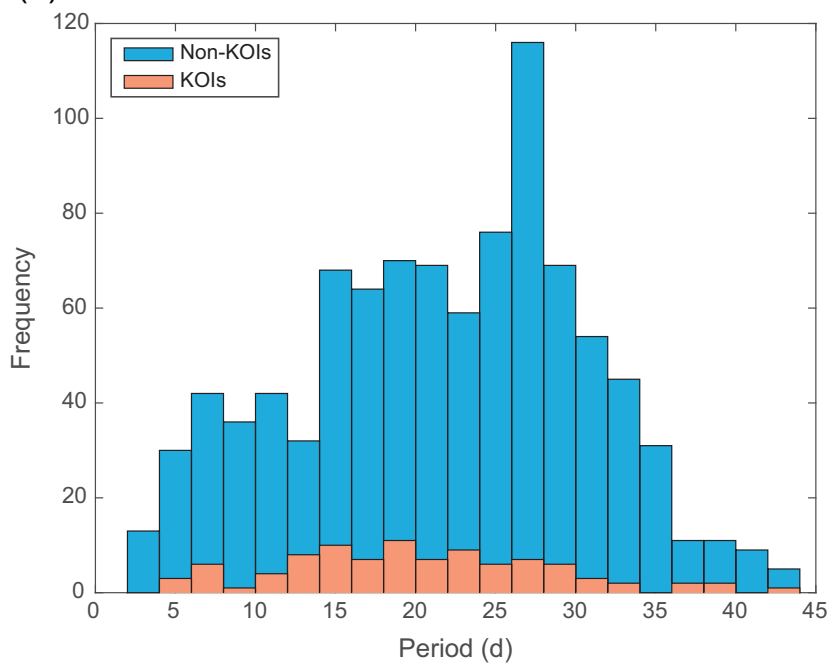

(b)

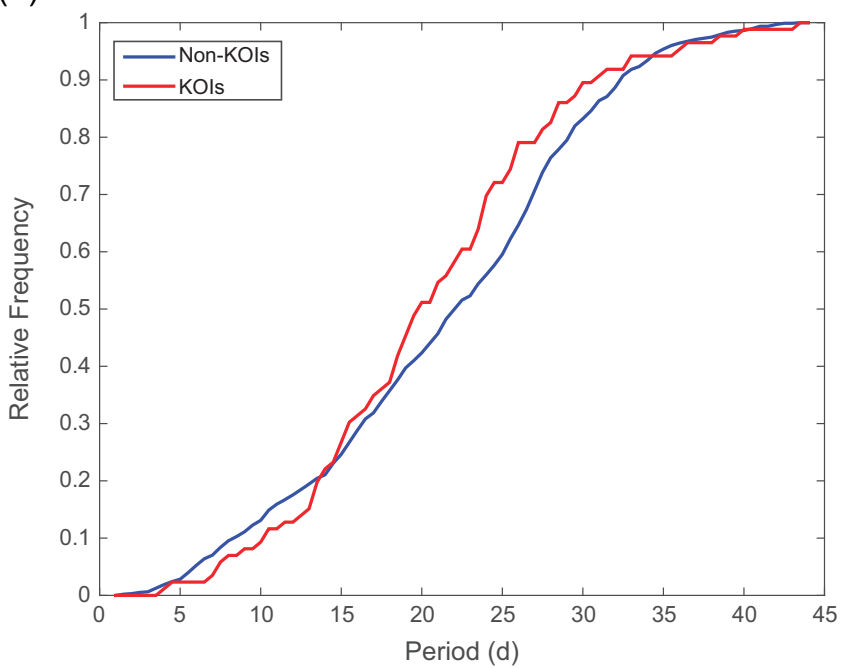

Fig. 8. (a) Compares histograms for the period distributions for the KOI and non-KOI samples, (b) CFDs for these cases and further compares Jupiter-hosting systems to the rest of the KOI distribution. A K-S test shows that the period distributions for the two cases (KOI and non-KOI) differ $(p=0.01$ ), with the planet-hosting sample showing a tendency to shorter periods, but is unable (with $p=0.06$ ) to discern any statistically significant difference between the Jupiter host population and the remainder of the KOI population.

(a)

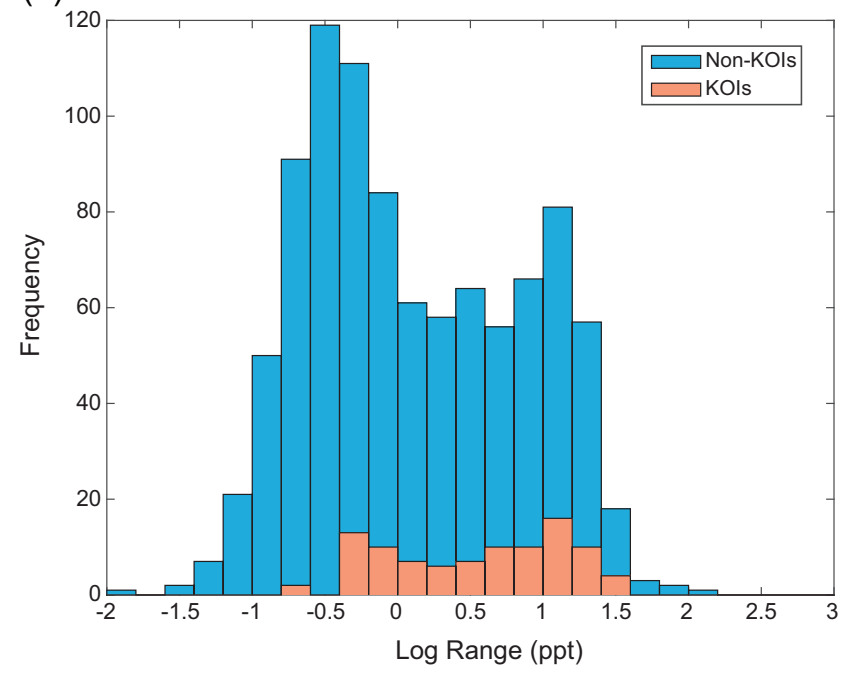

(b)

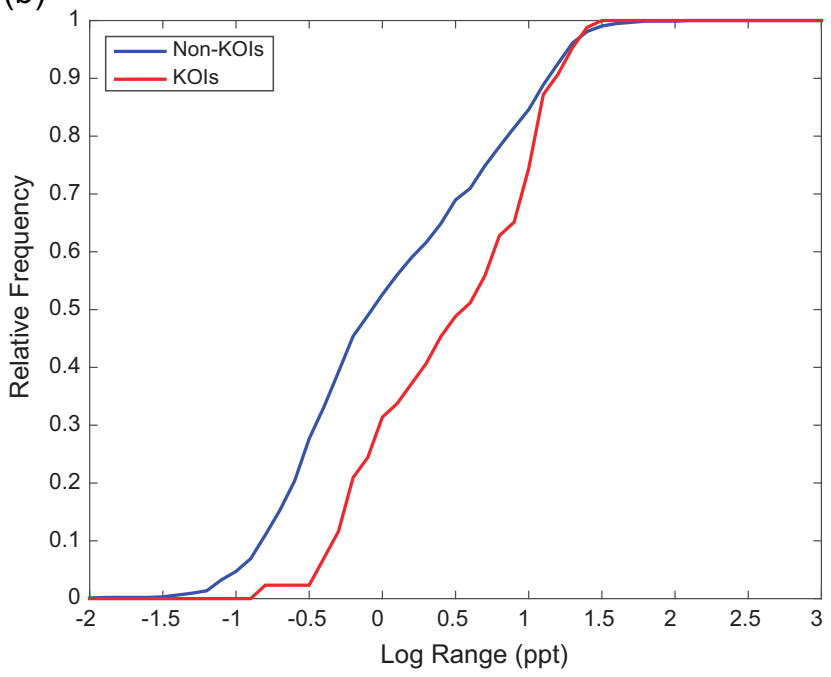

Fig. 9. (a) Compares histograms for the range distributions for the KOI and non-KOI samples, (b) CFDs for the two cases. The KOIs appear systematically more active than the non-KOIs, which is confirmed by statistical testing (K-S $\left.p<10^{-6}\right)$. We discuss our interpretation of this difference in Section 3.3.

with periods shorter than this, photometric activity levels (as characterized by the Range parameter) have a weak correlation with rotational period. However, at longer periods, the relationship vanishes and instead, a large variation in photometric activity level is observed for stars with similar rotation periods. We note that the dispersion observed in $R$ at long periods, more than two orders of magnitude, is considerably larger than one would expect from variations in the solar cycle or due to the range of projected stellar inclination in the sample, though both effects certainly account for a portion of the activity dispersion seen.

We next address the question of whether the level of photometric variability observed in the Sun is representative of that seen in solar-like stars. To do so, we examine the range parameter $R$ for all stars with measured rotation periods greater than 20 days and compare to the solar $R$ we previously derived from the Virgo data set, correcting as described in Section 3.3. Our resulting sample of 219 solar analogs has a mean rotation period of $27.9 \pm 4.9 \mathrm{~d}$, consistent (at the $1 \sigma$ level) with the solar rotation period. The vertical lines in Figure 11 indicate our measured high and low activity states for the Sun. From the figure, it is clear that the solar photometric activity level is comparable to the range of values seen from solar analogs. In fact, $40 \%$ of the range values for our sample lie between the active and inactive solar values from our Virgo data set, which is consistent with the earlier result of García et al. (2014) based on a much smaller data set. In this sense at least, the Sun is a good solar analog. 


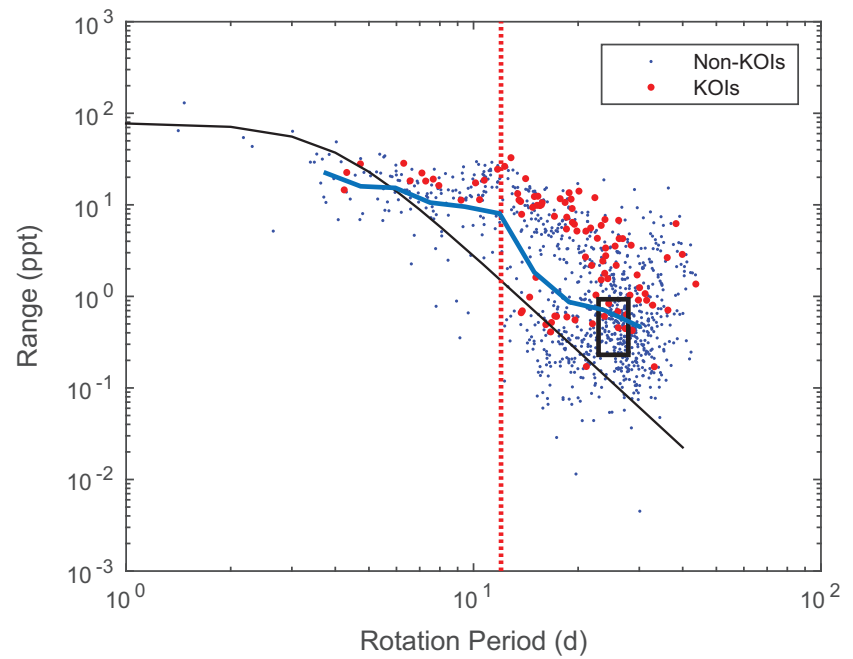

Fig. 10. The relation between photometric range $R$ and rotational period for both non-KOIs (blue) and KOIs (red circles) in our sample. The black line indicates the relationship posited by Hartman et al. (2009). The observed rotation-activity relation appears to have a break corresponding to periods of around $P=12 \pm 2 \mathrm{~d}$, illustrated by the dotted vertical line. Solar analogs with periods shorter than $\sim 12 \mathrm{~d}$ display a weak correlation with rotational period, but those with longer periods show pronounced scatter. This break is emphasized by the blue solid line, corresponding to the mean values of $\log R$ for bins of $0.1 \mathrm{dex}$ in $\log P$ (non-KOI targets only). The rectangle illustrates the position of the Sun on the diagram, with its size encoding uncertainties in the solar values derived from Virgo data as part of this work.

\subsection{Implications for gyrochronology}

The decline of rotation period with time on the main sequence was first noted by Skumanich (1972), but see also Kraft (1967) for an earlier look, who found that both chromospheric activity and rotation were proportional to $\tau^{-1 / 2}$. Over the succeeding decades a dependence of color (acting as a proxy for mass) was discovered and described by a variety of authors (Noyes et al. 1984; Kawaler 1988), the term "gyrochronology" was introduced by Barnes $(2003,2007)$, who fit a function of age and color to open cluster observations. More recently, the situation has become more complicated, with authors pointing out that cluster stars, field stars, and Kepler asteroseismology targets cannot be described by the same spindown function (Angus et al. 2015; Kovács 2015; Reinhold \& Gizon 2015; though see Epstein \& Pinsonneault 2014 and Barnes et al. 2016 for dissenting views). In particular, it appears that field stars may spin down following different relationships from those derived based on extrapolation of results from the (younger) open clusters (van Saders et al. 2016).

Here we examine this conclusion using our sample. Since our stars are carefully selected from a narrow range of physical parameters, the importance of the color term in any gyrochronological relationship should be minimized as we anticipate that its contribution will be similar for all stars. This effectively reduces the period-color-age relation to a simple period-age one. We made use of the TRILEGAL code (Girardi et al. 2005) to simulate the Kepler field, and sampled the result using the same criteria we used for the actual Kepler data, obtaining 1,815 solar analogs (comparable to the number in our initial sample). We then used stellar ages from the TRILEGAL simulation combined with rotation periods as predicted using several gyrochronology relations to produce

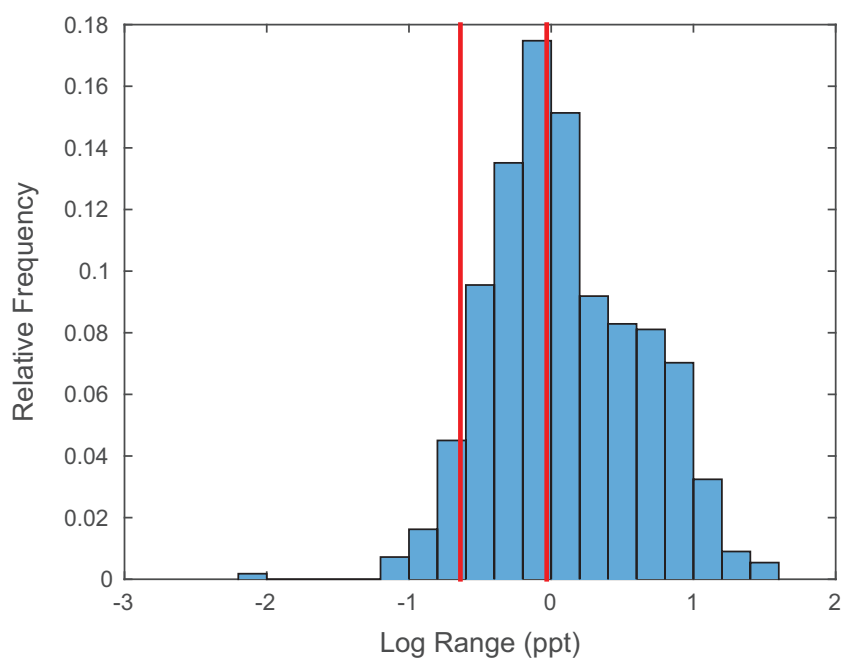

Fig. 11. The distribution of the photometric range parameter $R$ for all 219 non-KOI stars in our sample with $P>20$ d. The range has been corrected as described in Section 3.3. The vertical lines show the approximate positions of the Sun at high and low activity, as derived from Virgo data.

artificial rotation distributions to compare with our observed results. The uncertainty associated with specific TRILEGAL ages is itself somewhat uncertain, but appears to be roughly $\sim 30 \%$ (Miglio et al. 2013).

Figure 12 illustrates the outcomes of these comparisons; Figure 12a shows our observed result, while Figures $12 \mathrm{~b}-12 \mathrm{~d}$ show rotational period distributions obtained using gyrochronology relations from (Barnes 2007; Mamajek \& Hillenbrand 2008; Angus et al. 2015). While the general shape of the distribution for $P<20 \mathrm{~d}$ is successfully reproduced by all relations, none of the gyrochronology relations predict the presence of the observed tail extending to $P>30 \mathrm{~d}$, though Barnes (2007) and Mamajek \& Hillenbrand (2008) do somewhat better in that sense. The large dispersion in rotation rates may reflect the survival of such a dispersion in initial conditions, or perhaps an as-yet-unaccounted-for parameter in gyrochronology relations at large ages (where such relations are notoriously poorly constrained). In any case, we concur with the conclusions of recent authors Kovács (2015) and Angus et al. (2015), as well as references therein, that gyrochronology should be used with due care for older field stars.

\subsection{Obliquity}

As noted above, there appears to be a systematic difference between the photometric range of the KOI and non-KOI samples, with the former showing activity levels $\sim 0.3 \mathrm{dex}$ higher than the latter. This effect is unlikely to be physical in nature (i.e., caused by the presence of planets due to planet-star interactions) simply because based on published planet occurrence rates (Fressin et al. 2013) we anticipate that most of the non-KOI stars also host planets but are simply aligned in such a way as to make them undetectable by transits. Instead, we propose that the apparently higher photometric activity seen in the KOI systems is simply the artifact of a selection effect for stellar inclination, as previously suggested by Mazeh et al. (2015) and others.

If planetary orbits are closely aligned with the stellar rotational plane, so that orbital obliquities $\chi$ are small, then 
(a)

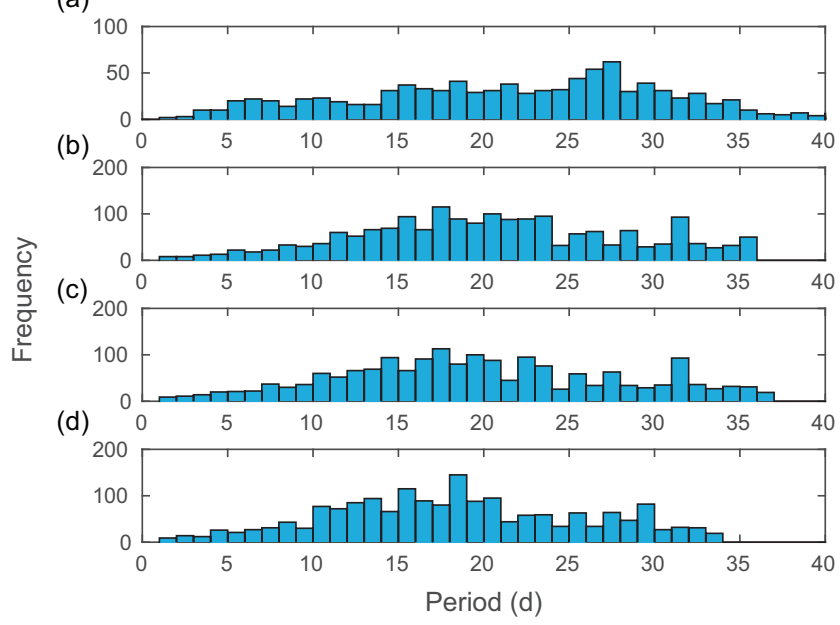

Fig. 12. (a) The distribution of measured rotation periods for the non-KOIs in our sample, (b-d) predicted distributions based on TRILEGAL population synthesis for the Kepler field combined with gyrochronology relations from Barnes (2007) (b), Mamajek \& Hillenbrand (2008) (c), and Angus et al. (2015) (d). All of the gyrochronology relations, when applied to the TRILEGAL sample, underpredict the number of slow rotators in the sample.

the KOI sample will tend to have stellar inclinations close to $90^{\circ}$ to the line of sight. In this case, starspots will transit close to the center of the disk and their effect on photometric variability will be at its maximum. The non-KOI sample, on the other hand, will contain a randomly-distributed range of stellar inclinations, and will on average show lower apparent photometric variability even if the stars themselves are similar to those in the KOI sample.

To understand this argument more readily, consider the case where all spots on all stars are the same size and occur on the stellar equator. In that vastly oversimplified case, the maximum photometric amplitude $R$ seen for any given star is solely a function of the inclination of the stellar rotational axis to the line of sight. Stars with inclinations near $90^{\circ}$ (equator-on) will have large photometric amplitudes, while those with inclinations near $0^{\circ}$ will have small amplitudes. For any randomly selected group of stars (i.e., the non-KOI sample in our case), the observed distribution (and mean) of the photometric range $R$ for that group will then simply reflect the probability distribution of stellar inclinations. If the distribution of orbital obliquities is random as well, so that there is no particular tendency for planetary orbits to align with the stellar rotational equator, the distribution (and mean) of the photometric range for stars with planets would look similar to that of stars without planets.

The KOI sample, however, is not randomly selected. The fact that we see transits in this sample tells us that the obliquity projected relative to our line of sight must be near zero, or transits would not be visible at all. If there were no correlation between the obliquity and the inclination, then the KOI sample and non-KOI sample photometric range distributions would look similar. However, if there is such a correlation, that would imply that stars with transits also have inclinations close to $90^{\circ}$, and thus would tend to appear more photometrically active because their spots would pass close to disk center.

The question we try to address is: how closely aligned on average is the orbital obliquity to the stellar rotational axis?
In the simple case outlined above (spots on all stars the same size and on the equator), there would be a unique relationship between photometric amplitude and obliquity for each individual star in the sample. However, real stars do not have spots that are all the same size and that are all located on the stellar equator. We try to address this by Monte Carlo sampling from a solar model population of spot distributions and randomized stellar inclinations; comparison of the mean photometric ranges expected from a range of different obliquities then allows us to coarsely estimate the obliquity distribution.

To model the starpot latitudinal distribution (the butterfly diagram), we use the Chang (2012) and Hathaway (2011) model (see also Santos et al. 2015) where the latitudinal distribution of spots is given by

$$
L_{\mathrm{S}}=L_{0} \exp -\left(\frac{t-t_{0}}{7.5}\right)
$$

for $t$ in years from the start of the cycle and $L_{0}=28^{\circ}$. For each model in our Monte Carlo simulation, we generated a random time in the 11-year activity cycle, used this to generate $L_{\mathrm{S}}$, and modified $L_{\mathrm{S}}$ by a convolution with the Ivanov \& Miletsky (2011) single-Gaussian description (with width $\sigma_{\mathrm{L}}$ ) of the sunspot formation zone. A single spot was then placed on the star and the stellar inclinations sampled among $[0,90]$ using the usual probability distribution.

$$
f(\psi)=\sin \psi \text {. }
$$

The photometric range $R$ was then estimated using the commonly-used photometric sunspot index or PSI (Froehlich et al. 1994),

$$
\mathrm{PSI}=\mu\left(\frac{3 \mu+2}{4}\right) \times a_{\mathrm{S}} \times \alpha .
$$

Here $\mu=\cos \theta$ in the usual sense, where $\theta$ is the angle relative to the local surface normal. The PSI is derived assuming Eddington limb darkening for both spot and photosphere; $a_{\mathrm{S}}$ is the sunspot covering fraction for the stellar disk, while $\alpha$ is the contrast between spot and photosphere. As PSI is essentially a way of quantifying the effect of sunspots on the total solar irradiance by estimating the photometric effect caused by spots of a given area, temperature, and location on the solar disk, it produces a result which is directly comparable (within some scaling factor) to our range parameter $R$. In our case, since we take the ratio of the range parameter between the two distributions, the exact values of $a_{\mathrm{S}}$ and $\alpha$ are irrelevant. While this is an oversimplified model, which in particular neglects the influence of plage (see, e.g. Shapiro et al. 2014 for a detailed discussion) and the presence of multiple spot groups, it is adequate for our purposes here. Figure 13a shows the average PSI calculated as a function of stellar inclination based on our model.

We consider stars with periods greater than 12 days to be solar-like in terms of their anticipated latitudinal distribution of spots (see Buzasi 1997; Schuessler \& Solanki 1992 for further discussion and justification for this assumption), and the observed distribution of photometric range $R$ for this sample is shown in Figure 14a for both KOIs and non-KOIs. We adopt the simplest possible model for mapping from the random (non-KOI) distribution to the biased (KOI) one, which is a linear map, so that

$$
\bar{R}_{\mathrm{KOI}}=\beta \bar{R}_{\text {non-KOI }} .
$$


(a)

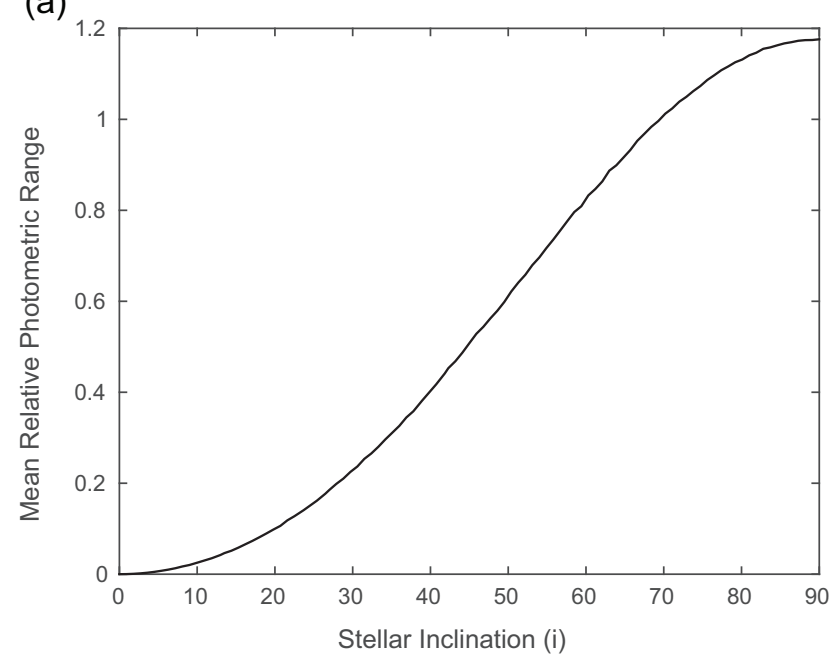

(b)

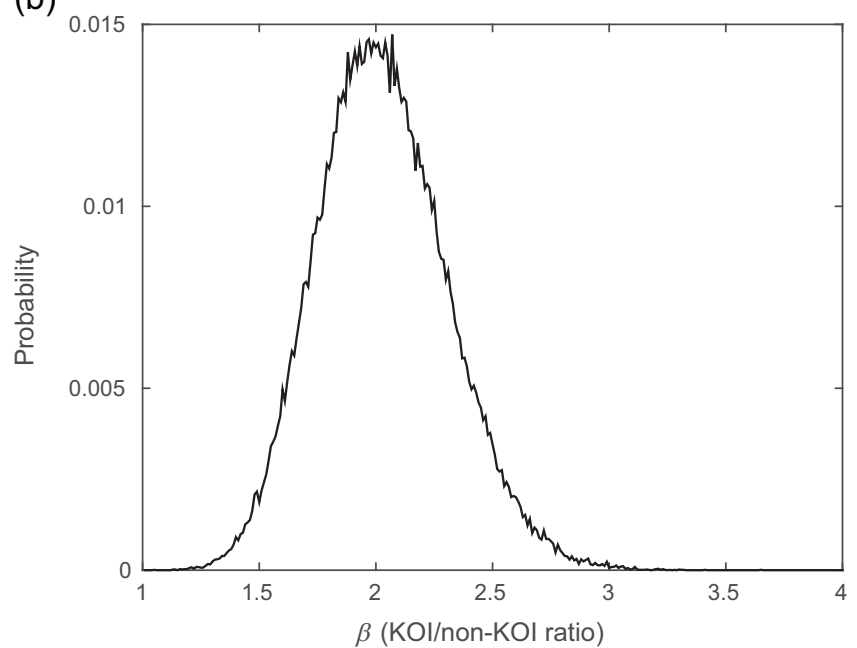

Fig. 13. (a) The average PSI calculated from our Monte Carlo simulation for spotted solar analogs, as described in the text, (b) the bootstrapped distribution of the $\beta$ parameter, the ratio of the mean observed range for KOIs relative to the non-KOI sample.

(a)

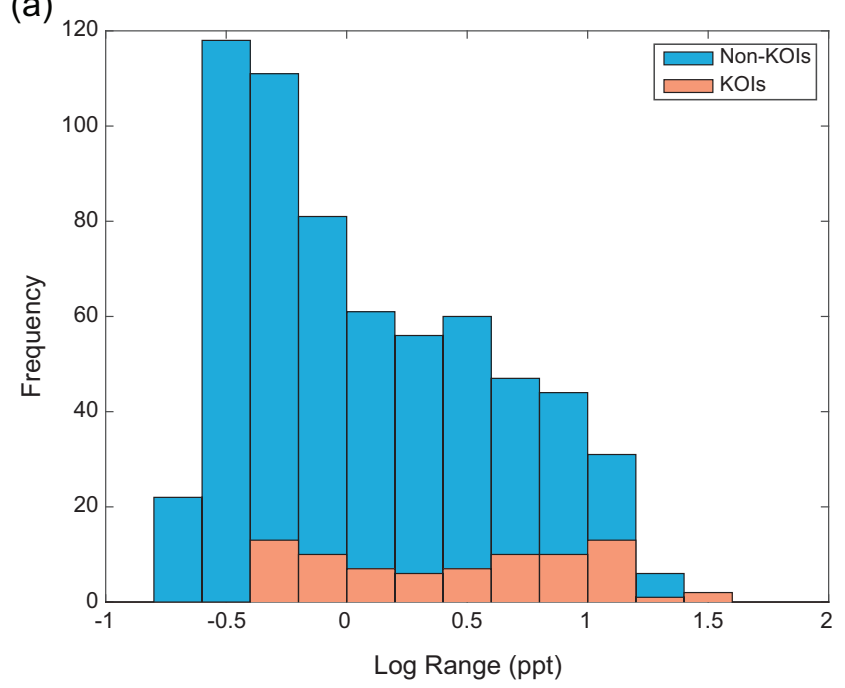

(b)

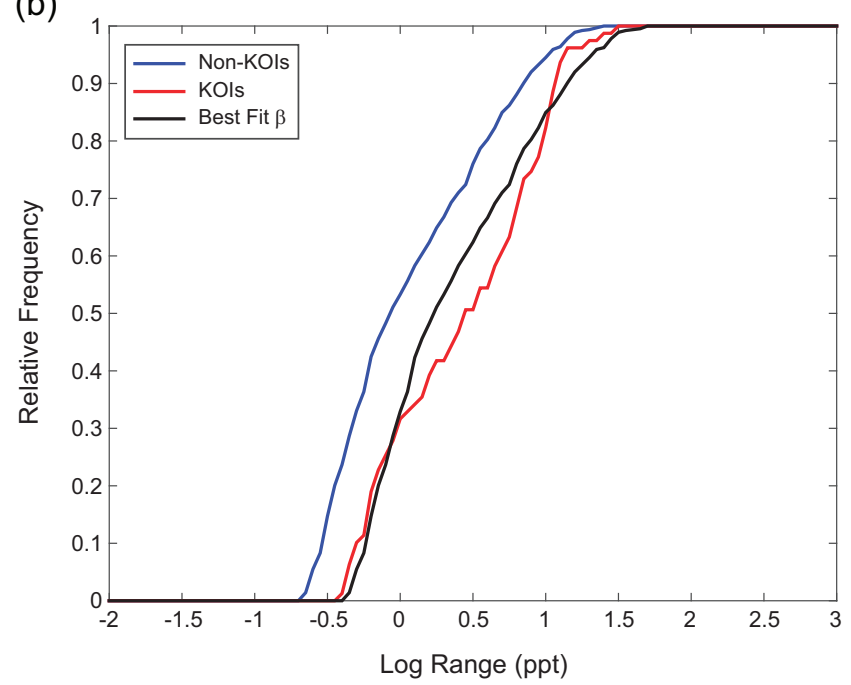

Fig. 14. (a) Compares histograms for the range distributions for the KOI and non-KOI samples with periods restricted to $P>12 \mathrm{~d}$, (b) $\mathrm{CFDs}$ for these cases and the best-fit model with $\beta=2.00$ (see text).

Figure $14 \mathrm{~b}$ shows the CDFs for the two distributions, as well as the CDF for the model with the best-fit value $\beta=2.00_{-0.28}^{+0.26}$; errors were estimated by a bootstrapping procedure performed on $\beta$ and illustrated in Figure $13 \mathrm{~b}$. We adopted a flat obliquity distribution in which the obliquity $\chi$ could take on any value between 0 and some maximum value $\chi_{\max }$, and performed $10^{6}$ Monte Carlo simulations of spots to determine the value of $\bar{\chi}$ consistent with the observed ratio of range distributions. Our resulting mean obliquity estimate is $\bar{\chi}=6_{-6}^{+5^{\circ}}$, implying that the majority of observed planetary systems around solar-like stars have small orbital inclinations relative to the stellar equator. The solar system, with an obliquity of roughly $7^{\circ}$ between the invariable plane and the solar equator, thus appears in this sense to be a good model for most exoplanet systems hosted by solar analogs. We note, however, that systematic errors are not treated here and are difficult to quantify.

\section{Conclusions}

We examined light curves for a large uniform sample of solar analogs in the Kepler field, using a simple but well-justified technique to extract rotational periods and photometric variability. Our approach allowed us to obtain rotational periods for less-variable stars than previous studies, at the expense of some precision in the resulting periods. The sample consisted of both planet-hosting stars (KOIs) and those not known to possess planets; the former with transits removed from the time series. The two subsets have similar ranges and distributions in $T_{\text {eff }}$ and metallicity $[\mathrm{Fe} / \mathrm{H}]$.

We measured similar distributions of rotation periods $P$ in both samples, with a distribution peaking around $20 \mathrm{~d}$ in each case, a result consistent with that seen by Ceillier et al. (2016), who compared a much smaller sample of planet hosts and non-hosts. The activity levels, characterized by the range parameter $R$ (essentially the photometric difference between 
the 5th and 95th percentile of the light curve), do show statistically significant differences between the two populations, with the KOIs appearing some $\sim 0.3$ dex more active overall. Both show similar patterns of activity decay with rotational period, with a weak relationship visible for periods less than about 12 days. For longer periods, the period-activity relation becomes much less clear and is characterized by a high degree of scatter. We compared solar activity levels derived from Virgo $g+r$ data at both solar minimum and maximum to our sample and concluded that the solar photometric range is comparable to that of the majority of solar analogs with rotational periods greater than 20 days.

Gyrochronology, the use of lower main sequence rotation rates as clocks to determine stellar ages, holds great promise, particularly for dating field stars too faint for asteroseismology. Unfortunately, in recent years, the hope of a single universal function that describes spindown for all stars on the lower main sequence has been receding, with various authors (Angus et al. 2015) noting that different functions appear to be required to describe the behavior of different populations. Brown (2014) succeeds in fitting the existing cluster data, but at the expense of invoking a probabilistic "metastable" dynamo that would likely render the useful application of gyrochronology to individual field stars difficult at best. The advantage of our test population for examining this issue comes from a combination of the large number of available rotation periods and the fact that all are (within uncertainties) the same color, thus removing the impact of the $f(B-V)$ term typically appearing in gyrochronology relations. Our conclusion is that none of the existing models fit well, particularly failing to match the observed number of slow rotators, and thus that the gyrochronology conundrum for older field stars is a real one.

We also used our data set to examine the obliquity of planetary systems hosted by solar analogs, under the presumption that the observed difference in activity levels between the KOI and non-KOI samples was due to a selection effect caused by the increased likelihood that systems with detected transiting planets were also observed with rotational inclinations near $90^{\circ}$. A simple model resulted in the estimated mean obliquity of $\bar{\chi}=6_{-6}^{+5^{\circ}}$. While the systematic error in this determination is likely large, it nonetheless suggests that the typical planetary system around a solar analog has low obliquity, as seen in our solar system. Other authors (Winn et al. 2010; Winn \& Fabrycky 2015) have suggested that systems involving lowmass hot Jupiters, particularly those hosted by hot stars, are frequently characterized by large obliquities, perhaps due to the lack of tidal dissipation in systems with thin convection zones (though see Rogers \& Lin 2013 for a theoretical discussion of this model). While our data set does not enable us to address that particular question, the observed difference between the two types of systems clearly has ramifications for models of planetary system formation and evolution.

While the Kepler mission is no more, the spacecraft itself continues in its new $\mathrm{K} 2$ identity to make stellar observations in new fields of view (Howell et al. 2014). Although the maximum dwell time on a single field of about 90 days limits the ability of $\mathrm{K} 2$ to detect planets in the habitable zone, this disadvantage is offset by the ability of the mission to observe a broader range of objects than was possible with Kepler. We plan to observe solar analogs in the K2 fields to both extend the current work, to search for systematic differences between different stellar populations, and to build a catalog of solar analogs for future work.
Acknowledgements. All authors would like to thank Lindsey Carboneau and Carly Hessler for useful discussions. DLB gratefully acknowledges the support of the Whitaker Center for STEM Education at Florida Gulf Coast University. Data used in the preparation of this paper were derived from NASA's Kepler mission and obtained through the Mikulski Archive for Space Telescopes (MAST) at the Space Telescope Science Institute, and from NASA's SOHO mission archive. The authors and editor also acknowledge useful comments from Mark Giampapa and an anonymous referee, which significantly improved the presentation of this paper.

\section{References}

Adibekyan, V.Z., P. Figueira, N.C. Santos, A.A. Hakobyan, S.G. Sousa, et al. Kinematics and chemical properties of the Galactic stellar populations. The HARPS FGK dwarfs sample. $A \& A, \mathbf{5 5 4}$, A44, 2013, DOI: 10.1051/0004-6361/201321520.

Affer, L., G. Micela, F. Favata, and E. Flaccomio. The rotation of field stars from CoRoT data. Mon. Not. R. Astron. Soc., 424, 11-22, 2012, DOI: 10.1111/j.1365-2966.2012.20802.x.

Aigrain, S., J. Llama, T. Ceillier, M.L.D. Chagas, J.R.A. Davenport, et al. Testing the recovery of stellar rotation signals from Kepler light curves using a blind hare-and-hounds exercise. Mon. Not. R. Astron. Soc., 450, 3211-3226, 2015, DOI: 10.1093/mnras/stv853.

Akeson, R.L., X. Chen, D. Ciardi, M. Crane, J. Good, et al. The NASA exoplanet archive: data and tools for exoplanet research. Publ. Astron. Soc. Pac., 125, 989-999, 2013, DOI: $10.1086 / 672273$.

Angus, R., S. Aigrain, D. Foreman-Mackey, and A. McQuillan. Calibrating gyrochronology using Kepler asteroseismic targets. Mon. Not. R. Astron. Soc., 450, 1787-1798, 2015, DOI: $10.1093 / \mathrm{mnras} / \mathrm{stv} 423$.

Baliunas, S., and R. Jastrow. Evidence for long-term brightness changes of solar-type stars. Nature, 348, 520-523, 1990, DOI: $10.1038 / 348520 \mathrm{a} 0$.

Baliunas, S.L., R.A. Donahue, W.H. Soon, J.H. Horne, J. Frazer, et al. Chromospheric variations in main-sequence stars. Astrophys. J., 438, 269-287, 1995, DOI: 10.1086/175072.

Barnes, S.A. On the rotational evolution of solar- and late-type stars, its magnetic origins, and the possibility of stellar gyrochronology. Astrophys. J., 586, 464-479, 2003,

DOI: $10.1086 / 367639$

Barnes, S.A. Ages for illustrative field stars using gyrochronology: viability, limitations, and errors. Astrophys. J., 669, 1167-1189, 2007, DOI: $10.1086 / 519295$.

Barnes, S.A., J. Weingrill, D. Fritzewski, K.G. Strassmeier, and I. Platais. Rotation periods for cool stars in the 4 Gyr old open cluster M67, the solar-stellar connection, and the applicability of gyrochronology to at least solar age. Astrophys. J., 823, 16, 2016, DOI: 10.3847/0004-637X/823/1/16.

Basri, G., L.M. Walkowicz, N. Batalha, R.L. Gilliland, J. Jenkins, et al. Photometric variability in Kepler target stars. II. An overview of amplitude, periodicity, and rotation in first quarter data. Astron. J., 141, 20, 2011,

DOI: $10.1088 / 0004-6256 / 141 / 1 / 20$.

Bastien, F.A., K.G. Stassun, G. Basri, and J. Pepper. An observational correlation between stellar brightness variations and surface gravity. Nature, 500, 427-430, 2013,

DOI: 10.1038/nature12419.

Borucki, W.J., D. Koch, G. Basri, N. Batalha, T. Brown, et al. Kepler planet-detection mission: introduction and first results. Science, 327, 977, 2010, DOI: 10.1126/science.1185402.

Brown, T.M. The metastable dynamo model of stellar rotational evolution. Astrophys. J., 789, 101, 2014, DOI: $10.1088 / 0004-637 \mathrm{X} / 789 / 2 / 101$

Brown, T.M., D.W. Latham, M.E. Everett, and G.A. Esquerdo. Kepler Input Catalog: photometric calibration and stellar classification. Astron. J., 142, 112-130, 2011, DOI: $10.1088 / 0004-6256 / 142 / 4 / 112$ 
Buzasi, D.L. Polar magnetic activity and spin-down on the lower main sequence. Astrophys. J., 484, 855-861, 1997.

Ceillier, T., J. van Saders, R.A. García, T.S. Metcalfe, O. Creevey, et al. Rotation periods and seismic ages of KOIs - comparison with stars without detected planets from Kepler observations. Mon. Not. R. Astron. Soc., 456, 119-125, 2016,

DOI: $10.1093 / \mathrm{mnras} / \mathrm{stv} 2622$.

Chang, H.-Y. Bimodal distribution of area-weighted latitude of sunspots and solar North-South asymmetry. New Astron., 17, 247-253, 2012, DOI: 10.1016/j.newast.2011.07.016.

Coughlin, J.L., S.E. Thompson, S.T. Bryson, C.J. Burke, D.A. Caldwell, et al. Contamination in the Kepler field. Identification of 685 KOIs as false positives via ephemeris matching based on Q1-Q12 data. Astron. J., 147, 119, 2014, DOI: $10.1088 / 0004-6256 / 147 / 5 / 119$.

Datson, J., C. Flynn, and L. Portinari. Spectroscopic study of solar twins and analogues. $A \& A, \mathbf{5 7 4}, \mathrm{A} 124,2015$, DOI: $10.1051 / 0004-6361 / 201425000$

do Nascimento Jr., R.A. García, J.-D., S. Mathur, F. Anthony, S.A. Barnes, et al. Rotation periods and ages of solar analogs and solar twins revealed by the Kepler Mission. Astrophys. J., 790, L23, 2014, DOI: 10.1088/2041-8205/790/2/L23.

Epstein, C.R., and M.H. Pinsonneault. How good a clock is rotation? The stellar rotation-mass-age relationship for old field stars. Astrophys. J., 780, 159, 2014,

DOI: $10.1088 / 0004-637 \mathrm{X} / 780 / 2 / 159$

Fressin, F., G. Torres, D. Charbonneau, S.T. Bryson, J. Christiansen, C.D. Dressing, J.M. Jenkins, L.M. Walkowicz, and N.M. Batalha. The false positive rate of Kepler and the occurrence of planets. Astrophys. J., 766, 81, 2013, DOI: 10.1088/0004-637X/766/2/81.

Froehlich, C., J.M. Pap, and H.S. Hudson. Improvement of the photometric sunspot index and changes of the disk-integrated sunspot contrast with time. Sol. Phys., 152, 111-118, 1994, DOI: $10.1007 / \mathrm{BF} 01473192$.

Gafeira, R., C.C. Fonte, M.A. Pais, and J. Fernandes. Temporal evolution of sunspot areas and estimation of related plasma flows. Sol. Phys., 289, 1531-1542, 2014,

DOI: $10.1007 / \mathrm{s} 11207-013-0440-3$.

García, R.A., T. Ceillier, D. Salabert, S. Mathur, J.L. van Saders, et al. Rotation and magnetism of Kepler pulsating solar-like stars. Towards asteroseismically calibrated age-rotation relations. $A \& A$, 572, A34, 2014, DOI: 10.1051/0004-6361/201423888.

Giampapa, M.S., J.C. Hall, R.R. Radick, and S.L. Baliunas. A survey of chromospheric activity in the solar-type stars in the open cluster M67. Astrophys. J., 651, 444-461, 2006, DOI: $10.1086 / 507624$.

Gilliland, R.L., W.J. Chaplin, E.W. Dunham, V.S. Argabright, W.J. Borucki, et al. Kepler mission stellar and instrument noise properties. Astrophys. J. Supp., 197, 6, 2011, DOI: $10.1088 / 0067-0049 / 197 / 1 / 6$

Girardi, L., M.A.T. Groenewegen, E. Hatziminaoglou, and L. da Costa. Star counts in the Galaxy. Simulating from very deep to very shallow photometric surveys with the TRILEGAL code. $A \& A$, 436, 895-915, 2005, DOI: 10.1051/0004-6361:20042352.

Hall, J.C., G.W. Lockwood, and B.A. Skiff. The activity and variability of the sun and sun-like stars. I. Synoptic $\mathrm{Ca}$ II $\mathrm{H}$ and $\mathrm{K}$ observations. Astron. J., 133, 862-881, 2007, DOI: $10.1086 / 510356$.

Hall, J.C., G.W. Henry, G.W. Lockwood, B.A. Skiff, and S.H. Saar. The activity and variability of the sun and sun-like stars. II Contemporaneous photometry and spectroscopy of bright solar analogs. Astron. J., 138, 312-322, 2009,

DOI: $10.1088 / 0004-6256 / 138 / 1 / 312$.

Hartman, J.D., B.S. Gaudi, M.J. Holman, B.A. McLeod, K.Z. Stanek, J.A. Barranco, M.H. Pinsonneault, S. Meibom, and J.S. Kalirai. Deep MMT transit survey of the open cluster M37 IV: limit on the fraction of stars with planets as small as $0.3 \mathrm{R}_{J}$. Astrophys. J., 695, 336-356, 2009,

DOI: $10.1088 / 0004-637 \mathrm{X} / 695 / 1 / 336$.
Hathaway, D.H. A standard law for the equatorward drift of the sunspot zones. Sol. Phys., 273, 221-230, 2011,

DOI: $10.1007 / \mathrm{s} 11207-011-9837-z$.

Howell, S.B., C. Sobeck, M. Haas, M. Still, T. Barclay, et al. The K2 mission: characterization and early results. Publ. Astron. Soc. Pac., 126, 398-408, 2014, DOI: 10.1086/676406.

Huber, D., V. Silva Aguirre, J.M. Matthews, M.H. Pinsonneault, E. Gaidos, et al. Revised stellar properties of Kepler targets for the quarter 1-16 transit detection run. Astrophysical Journal Supplement Series, 211, 2, 2014, DOI: $10.1088 / 0067-0049 / 211 / 1 / 2$

Ivanov, V.G., and E.V. Miletsky. Width of sunspot generating zone and reconstruction of butterfly. Sol. Phys., 268, 231-242, 2011, DOI: $10.1007 / \mathrm{s} 11207-010-9665-6$.

Kawaler, S.D. Angular momentum loss in low-mass stars. Astrophys. J., 333, 236-247, 1988, DOI: 10.1086/166740.

Kovács, G. Are the gyro-ages of field stars underestimated? $A \& A$, 581, A2, 2015, DOI: 10.1051/0004-6361/201525920.

Kraft, R.P. Studies of stellar rotation. V. The dependence of rotation on age among solar-type stars. Astrophys. J., 150, 551, 1967, DOI: $10.1086 / 149359$.

Lubin, D., D. Tytler, and D. Kirkman. Lithium abundance in solartype stars with low chromospheric activity: application to the search for maunder minimum analogs. Astrophys. J., 716, 766-775, 2010, DOI: 10.1088/0004-637X/716/1/766.

Lubin, D., D. Tytler, and D. Kirkman. Frequency of Maunder minimum events in solar-type stars inferred from activity and metallicity observations. Astrophys. J., 747, L32, 2012, DOI: 10.1088/2041-8205/747/2/L32.

Mamajek, E.E., and L.A. Hillenbrand. Improved age estimation for solar-type dwarfs using activity-rotation diagnostics. Astrophys. J., 687, 1264-1293, 2008, DOI: 10.1086/591785.

Mamajek, E.E., A. Prsa, G. Torres, P. Harmanec, M. Asplund, et al. IAU 2015 resolution B3 on recommended nominal conversion constants for selected solar and planetary properties. arXiv: 1510.07674, 2015.

Mazeh, T., H.B. Perets, A. McQuillan, and E.S. Goldstein. Photometric amplitude distribution of stellar rotation of KOIs indication for spin-orbit alignment of cool stars and high obliquity for hot stars. Astrophys. J., 801, 3, 2015, DOI: $10.1088 / 0004-637 \mathrm{X} / 801 / 1 / 3$.

McQuillan, A., T. Mazeh, and S. Aigrain. Rotation periods of 34,030 Kepler main-sequence stars: the full autocorrelation sample. Astrophysical Journal Supplement Series, 211, 24, 2014, DOI: $10.1088 / 0067-0049 / 211 / 2 / 24$.

Miglio, A., C. Chiappini, T. Morel, M. Barbieri, W.J. Chaplin, et al. Galactic archaeology: mapping and dating stellar populations with asteroseismology of red-giant stars. Mon. Not. R. Astron. Soc., 429, 423-428, 2013, DOI: 10.1093/mnras/sts345.

Nielsen, M.B., L. Gizon, H. Schunker, and C. Karoff. Rotation periods of 12000 main-sequence Kepler stars: dependence on stellar spectral type and comparison with $\mathrm{v}$ sin i observations. $A \& A$, 557, L10, 2013, DOI: 10.1051/0004-6361/201321912.

Noyes, R.W., L.W. Hartmann, S.L. Baliunas, D.K. Duncan, and A.H. Vaughan. Rotation, convection, and magnetic activity in lower main-sequence stars. Astrophys. J., 279, 763-777, 1984, DOI: $10.1086 / 161945$.

Önehag, A., A. Korn, B. Gustafsson, E. Stempels, and D.A. Vandenberg. M67-1194, an unusually sunlike solar twin in M67. $A \& A, \mathbf{5 2 8}$, A85, 2011, DOI: 10.1051/0004-6361/201015138.

Pinsonneault, M.H., D. An, J. Molenda-Żakowicz, W.J. Chaplin, T.S. Metcalfe, and H. Bruntt. A revised effective temperature scale for the Kepler Input Catalog. Astrophysical Journal Supplement Series, 199, 30, 2012, DOI: 10.1088/0067-0049/199/2/30.

Porto de Mello, G.F., R. da Silva, L. da Silva, and R.V. de Nader. A photometric and spectroscopic survey of solar twin stars within 50 parsecs of the Sun. I. Atmospheric parameters and color similarity to the Sun. $A \& A, \mathbf{5 6 3}$, A52, 2014, DOI: $10.1051 / 0004-6361 / 201322277$. 
D. Buzasi et al.: Solar analogs in the Kepler field

Prša, A., N. Batalha, R.W. Slawson, L.R. Doyle, W.F. Welsh, et al. Kepler eclipsing binary stars. I. Catalog and principal characterization of 1879 eclipsing binaries in the first data release. Astron. $J ., 141,83,2011$, DOI: 10.1088/0004-6256/141/3/83.

Radick, R.R., G.W. Lockwood, B.A. Skiff, and S.L. Baliunas. Patterns of variation among sun-like stars. Astrophysical Journal Supplement Series, 118, 239-258, 1998, DOI: 10.1086/313135.

Reinhold, T., and L. Gizon. Rotation, differential rotation, and gyrochronology of active Kepler stars. $A \& A, \mathbf{5 8 3}$, A65, 2015, DOI: 10.1051/0004-6361/201526216.

Reinhold, T., A. Reiners, and G. Basri. Rotation and differential rotation of active Kepler stars. $A \& A, \mathbf{5 6 0}, \mathrm{A} 4,2013$, DOI: $10.1051 / 0004-6361 / 201321970$.

Rocha-Pinto, H.J., and W.J. Maciel. The metallicity distribution of G dwarfs in the solar neighbourhood. Mon. Not. R. Astron. Soc., 279, 447-458, 1996, DOI: 10.1093/mnras/279.2.447.

Rogers, T.M., and D.N.C. Lin. On the tidal dissipation of obliquity. Astrophys. J., 769, L10, 2013, DOI: $10.1088 / 2041-8205 / 769 / 1 / \mathrm{L} 10$.

Santos, A.R.G., M.S. Cunha, P.P. Avelino, and T.L. Campante. Spot cycle reconstruction: an empirical tool. Application to the sunspot cycle. $A \& A, \mathbf{5 8 0}, \mathrm{A} 62,2015$, DOI: $10.1051 / 0004-6361 / 201425299$.

Schuessler, M., and S.K. Solanki. Why rapid rotators have polar spots. $A \& A$, 264, L13-L16, 1992.

Shapiro, A.I., S.K. Solanki, N.A. Krivova, W.K. Schmutz, W.T. Ball, R. Knaack, E.V. Rozanov, and Y.C. Unruh. Variability of sun-like stars: reproducing observed photometric trends. $A \& A, \mathbf{5 6 9}, \mathrm{A} 38$, 2014, DOI: $10.1051 / 0004-6361 / 201323086$.

Skumanich, A. Time scales for CA II emission decay, rotational braking, and lithium depletion. Astrophys. J., 171, 565, 1972, DOI: $10.1086 / 151310$.
Smith, J.C., M.C. Stumpe, J.E. Van Cleve, J.M. Jenkins, T.S. Barclay, et al. Kepler presearch data conditioning II - a Bayesian approach to systematic error correction. Publ. Astron. Soc. Pac., 124, 1000-1014, 2012, DOI: 10.1086/667697.

Takeda, Y. Fundamental parameters and elemental abundances of 160 F-G-K stars based on OAO spectrum database. Publ. Astron. Soc. Jpn., 59, 335-356, 2007, DOI: 10.1093/pasj/59.2.335.

Takeda, Y., A. Tajitsu, S. Honda, S. Kawanomoto, H. Ando, and T. Sakurai. Detection of low-level activities in solar-analog stars from emission strengths of the Ca II 3934 line. Publ. Astron. Soc. Jpn., 64, 130, 2012, DOI: 10.1093/pasj/64.6.130.

van Saders, J.L., T. Ceillier, T.S. Metcalfe, V. Silva Aguirre, M.H. Pinsonneault, R.A. García, S. Mathur, and G.R. Davies. Weakened magnetic braking as the origin of anomalously rapid rotation in old field stars. Nature, 529, 181-184, 2016, DOI: 10.1038/nature16168.

Walkowicz, L.M., and G.S. Basri. Rotation periods, variability properties and ages for Kepler exoplanet candidate host stars. Mon. Not. R. Astron. Soc., 436, 1883-1895, 2013, DOI: $10.1093 / \mathrm{mnras} / \mathrm{stt} 1700$.

Winn, J.N., and D.C. Fabrycky. The occurrence and architecture of exoplanetary systems. Annu. Rev. Astron. Astrophys., 53, 409-447, 2015, DOI: 10.1146/annurev-astro-082214-122246.

Winn, J.N., D. Fabrycky, S. Albrecht, and J.A. Johnson. Hot stars with hot Jupiters have high obliquities. Astrophys. J., 718, L145-L149, 2010, DOI: 10.1088/2041-8205/718/2/L145.

Wright, J.T. Do we know of any Maunder minimum stars? Astron. J., 128, 1273-1278, 2004, DOI: 10.1086/423221.

Cite this article as: Buzasi D, Lezcano A \& Preston HL. Rotation, activity, and stellar obliquities in a large uniform sample of Kepler solar analogs. J. Space Weather Space Clim., 6, A38, 2016, DOI: 10.1051/swsc/2016033. 